\title{
THE COLOR-MAGNITUDE RELATION OF BULGES
}

\author{
NOBUNARI ITOH \\ Kiso Observatory, Institute of Astronomy, University of Tokyo \\ Mitake-mura, Kiso-gun, Nagano 397-01, Japan
}

AND

TAKASHI ICHIKAWA

Department of Astronomy, Tohoku University

Aoba-ku, Sendai 980, Japan

\section{Introduction}

One of the main aims with respect to the evolution of spiral galaxies is to clarify how the disk and bulge were formed. The study of bulges has usually been focused on how bulges resemble to elliptical galaxies. For the study of the evolution of elliptical galaxies, the color-magnitude relation (C-M relation) gives us a clue to understanding the formation and evolution of ellipticals. In fact, the tight C-M relation of ellipticals (Bower et al. 1992) was interpreted as the sequence of the metallicity difference (Kodama and Arimoto 1997). On the contrary, there are few observations for the C-M relation of bulges. On the analogy of elliptical galaxies, the C-M relation of bulges, if it exists, may be one of the most important clues to understanding the formation process of bulges. The main goal of the present study is to investigate the $\mathrm{C}-\mathrm{M}$ relation of bulges.

\section{Observations}

Observations were performed in Johnson $B, V$, and Kron-Cousins $R_{c}, I_{c}$ bands with a CCD camera attached to the prime focus of the $105 \mathrm{~cm}$ Schmidt telescope at the Kiso Observatory of the University of Tokyo. The field of view of the camera is $12.5^{\prime} \times 12.8^{\prime}$ with a resolution of $0.75^{\prime \prime} \times 0.75^{\prime \prime}$ per pixel. 


\section{Results}

We measure the colors of the bulges in the fan-shaped aperture opened along the minor axis since the contribution of disk light becomes lower and the dust extinction becomes less effective at the large distance from the galactic plane along the minor axis. The measured bulge colors are not intrinsic because there is the contribution of the disk color to the bulge color. We decompose each galaxy into a disk and a bulge and correct the observed bulge color using decomposition parameters. The detail procedure of the correction is mentioned by Itoh (1997).

We compare the bulge colors with the bulge magnitude. Figure 1 shows the plot of the bulge colors as a function of bulge magnitude. The bright bulges tend to be redder than the faint bulges. This tendency is similar to the color-magnitude relation of ellipticals. The regression, the scatter $(\sigma)$ and the correlation coefficient $(r)$ for each color are obtained with the least-square fitting. The results are

$$
\begin{aligned}
(B-I)_{o}= & -0.051( \pm 0.047) \cdot\left(M_{I, \text { bulge }}+20\right)+2.161( \pm 0.048), \\
& \sigma=0.075, r=-0.726 .
\end{aligned}
$$

It is interesting to compare the CM relation of the bulge with that of ellipticals. We can see in figure 1 that the slope of the bulge CM relation is consistent with that of elliptical within errors and that bulges tend to be redder than ellipticals with the same luminosity. The color offsets of the bulge from the elliptical at $M_{I}=-20 \mathrm{mag}$ are $\Delta(B-I)_{\text {bulge-elli. }}=0.09 \pm 0.08 \mathrm{mag}$.

In summary, bulges have the color-magnitude relation.

\section{Reference}

Bower, R.G., Lucey, J.R., and Ellis, R., (1992), MNRAS, 254, 589.

Itoh, N., (1997), Ph. D. thesis, University of Tokyo.

Kodama, T. and Arimoto, N., (1997) $A \& B$ A, 320, 41.

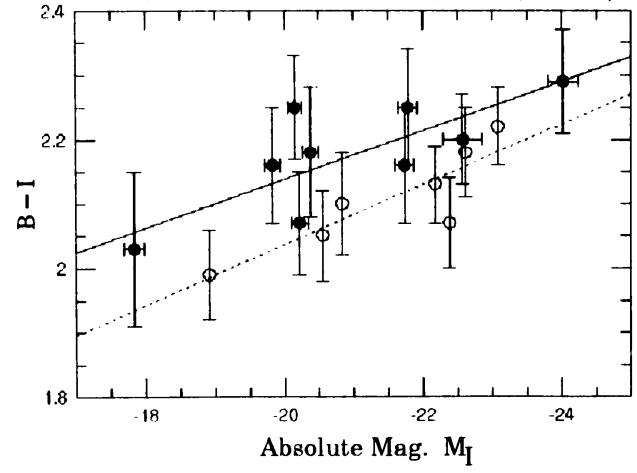

Figure 1

The color-magnitude relations of bulges and ellipticals.

Filled circles indicate bulges and open circles ellipticals.

The solid and dotted lines show regressions for bulge and ellipticals respectively. 